\title{
A novel use of the precordial Doppler for verification of central venous access
}

\author{
Mark Alexander Burbridge \\ Department of Anesthesiology, Perioperative and Pain Medicine, Stanford University School of Medicine, Stanford, \\ CA, USA
}

This communication discusses a case demonstrating a novel use of the precordial Doppler (Versatone ${ }^{\circledR}$, MedaSonics, USA) in verifying the intravenous placement of a central venous catheter. The precordial Doppler is otherwise commonly used during neurosurgical procedures to monitor for venous air embolism.

This case involves a 58-year-old morbidly obese female (body mass index $=58$ ) presenting for clipping of a ruptured aneurysm of the middle cerebral artery under mild hypothermia. Preoperatively, a 22-g upper extremity intravenous catheter was placed; however, three attempts were required owing to poor venous targets. After induction of anesthesia, a similar lack of appropriate venous targets was noted even with extensive ultrasound imaging in both upper and lower extremities. A large bore central line sheath (9 French, $10 \mathrm{~cm}$ sheath, Arrow International, USA) was therefore indicated. The surgical team required the right side of the neck to be sterile for possible clamping of the internal carotid artery, if aneurysm rupture necessitates gaining proximal control. The subclavian placement of the sheath was also deemed undesirable owing to the patient's body habitus. A right internal jugular attempt was made by the anesthesia resident, but an inadvertent carotid puncture produced a large hematoma that subsequently obscured the visualization of the internal jugular vein and carotid artery. The decision was then made to gain

Corresponding author: Mark Alexander Burbridge, M.D.

Department of Anesthesiology, Perioperative and Pain Medicine, Stanford University School of Medicine, 300 Pasteur Drive, Stanford, CA 94304, USA

Tel: 1-650-353-8484, Fax: 1-650-725-8052

Email: markburb@stanford.edu

ORCID: https://orcid.org/0000-0001-6765-5739

Received: August 29, 2018.

Accepted: September 3, 2018.

Korean J Anesthesiol 2019 February 72(1): 76-77

https://doi.org/10.4097/kja.d.18.00252 access to the femoral vein. Under ultrasound imaging, the right femoral vein was cannulated with the aforementioned sheath, and its placement was verified with a central venous pressure (CVP) of $5 \mathrm{cmH}_{2} \mathrm{O}$, a characteristic CVP waveform, and positive aspiration of dark, non-pulsatile blood. However, concern regarding migration of this line from the vein remained during this long procedure owing to the deep location of the vein $(7 \mathrm{~cm}$ below the surface of the skin) and presence of copious mobile adipose tissue that could possibly shift the line with positioning changes. Increased compliance of the patient's skin and subcutaneous tissues probably would have made it difficult to detect the line migrating out of the femoral vein otherwise.

To monitor the intravenous placement of the line, a precordial Doppler was positioned on the anterior aspect of the chest in the 2 nd intercostal space on the right side. Intermittent rapid flushes of $10 \mathrm{ml}$ of saline were administered during the procedure, resulting in the characteristic turbulent acoustic signal, thereby verifying the intravenous placement of the sheath. Just prior to aneurysm clipping, the intravenous placement of the line was again verified to aid in fluid and/or blood transfusion in the event of sudden massive blood loss. The line remained in the desired intravenous location during the procedure, and its position was again verified upon arrival to the intensive care unit post-operatively.

The correct placement of central venous lines can be verified by several techniques including radiography, central venous waveform analysis, and ultrasound techniques $[1,2]$. This novel technique has not been described previously, and can henceforth be considered as an acceptable technique to verify the initial placement and/or continuous placement of central venous lines.

(c) This is an open-access article distributed under the terms of the Creative Commons Attribution Non-Commercial License (http://creativecommons.org/ licenses/by-nc/4.0/), which permits unrestricted non-commercial use, distribution, and reproduction in any medium, provided the original work is properly cited. 


\section{References}

1. Abood GJ, Davis KA, Esposito TJ, Luchette FA, Gamelli RL. Comparison of routine chest radiograph versus clinician judgment to determine adequate central line placement in critically ill patients. J Trauma 2007; 63: 50-6.

2. Meggiolaro M, Scatto A, Zorzi A, Roman-Pognuz E, Lauro A, Passarella C, et al. Confirmation of correct central venous catheter position in the preoperative setting by echocardiographic "bubble-test". Minerva Anestesiol 2015; 81: 989-1000. 